\title{
Post-Flight Test Results of Seed Laser Module Subjected to Space Exposure
}

\author{
Narasimha S. Prasad* \\ NASA Langley Research Center, 5 N. Dryden St., MS 468, Hampton VA, 23681
}

\begin{abstract}
The objective of the Materials International Space Station Experiment (MISSE) is to study the performance of novel materials when subjected to the synergistic effects of the harsh space environment for several months. MISSE missions provide an opportunity for developing space qualifiable materials. Several laser and lidar components were sent by NASA Langley Research Center (LaRC) as a part of the MISSE 7 mission. The MISSE 7 module was transported to the international space station (ISS) via STS 129 mission that was launched on Nov 16, 2009. Later, the MISSE 7 module was brought back to the earth via the STS 134 that landed on June 1, 2011. The MISSE 7 module that was subjected to exposure in space environment for more than one and a half year included fiber laser, solid-state laser gain materials, detectors, and semiconductor laser diode. Performance testing of these components is now progressing. In this paper, the results of performance testing of a laser diode module sent by NASA Langley Research Center on MISSE 7 mission will be discussed. This paper will present the comparison of pre-flight and post-flight performance curves and discuss the effect of space exposure on the laser diode module. Preliminary findings on output power measurements show that the COTS laser diode characteristics did not undergo any significant performance degradation.
\end{abstract}

Keywords: MISSE 7, Space Qualification, Lidar components, International Space Station (ISS), STS-129, STS-134

\section{INTRODUCTION}

The goal of MISSE program is to evaluate the performance, stability, and long-term survivability of materials and components planned for use by NASA, Department of Defense (DOD), other federal agencies and private entities on future Low Earth Orbit (LEO), synchronous orbit, and interplanetary space missions. The study of combined effects of radiation, ultraviolet (UV) light and atomic oxygen in space due to long term exposures will help in developing space qualifiable elements for future space missions. In-situ space testing is critical since it is difficult and not economical to conduct these studies inside simulated terrestrial facilities. The development of new generations of materials will allow our nation to continue maintain technological superiority related to space endeavors.

NASA's Langley Research Center (LaRC), Hampton, Virginia has managed the MISSE projects till recently. NASA LaRC successfully completed MISSE 6 mission and supported follow-on MISSE 7 and 8 missions. Other NASA Centers participating in MISSE project include Glenn Research Center, Cleveland; Goddard Space Flight Center, Greenbelt, Maryland, Johnson Space Center, Houston, Texas, the Jet Propulsion Laboratory, Pasadena, California, and Marshall Space Flight Center, Huntsville, Alabama.. Several DOD and industry partners including the Boeing Company have been involved with this effort. MISSE program is a direct successor of the Mir Environmental Effects Payloads (MEEP) that were attached for over a year to the Mir Docking Module of the space station Mir between shuttle flights STS-76 and STS-86 [1].

\section{AN OVERVIEW OF THE MISSE PROGRAM}

MISSE is a series of experiments and so far, five MISSE missions have been successfully completed. More than 1500 samples have been tested on the MISSE project. Samples include chemicals, sensor devices, opto-mechanical elements, polymers, coatings, and biological materials and species, composites. The MISSE project has also provided

\footnotetext{
*narasimha.s.prasad@nasa.gov; Phone 757-864-9403; Fax 757-864-8828.
} 
educational opportunities for students. MISSEs 3 and 4 have transported approximately 8 million basil seeds for science experiments of children to stimulate interest in space science.

A suitcase shaped rugged box known as Passive Experiment Containers (PECs) is used to transport the selected materials to and from the ISS. PECs were originally developed and used by NASA's Langley Research Center, Hampton, Virginia, for ISS Phase I Risk Mitigation Program experiments conducted on the Russian Mir space station. Specific steps required for transporting materials include specimen preparation, fixing specimens inside a holder, integration of specimen holder on a tray, integration of trays in a PEC and integration of PEC inside a carrier for ease of transport. All these tasks are carried out in clean room environment.

The PEC is tested for survivability under launch conditions by subjecting it to appropriate shock and vibration environment. Subsequent to several months of space exposure, the PEC will be transported back to the Earth to undergo tests. The resulting test characteristics when compared with original characteristics before launch will provide an understanding of their survivability in space conditions. Furthermore, it will provide insights into required space qualification processes for future space applications.

During space walk, also known as extra-vehicular activity (EVA) (i.e., when an astronaut works outside of a spacecraft), the PECs are attached to the handrails or at a specific location that is exposed to space environment. Two possible mounting positions known as ram and wake are available. The materials in the ram side will be predominantly subjected to atomic oxygen environment where as those materials in the wake side will mostly undergo UV exposure. After exposure in space, MISSE PEC is retrieved in the same manner as it was deployed. The material samples are tested in laboratory conditions to see if they still possess their unique properties needed to complete space missions. MISSE PECs have active and passive detectors to give a time-history reading, or a reading of what happened to the materials at certain points in time. Back on the ground, tests will be conducted to determine the effects of its exposure for several months in space. The passive detectors report a cumulative measure of the following environments that the test specimens are exposed to namely UV exposure, atomic oxygen exposure, molecular contamination, tray temperature and man-made debris.

Terrestrial laboratories may provide limited environmental test conditions while in space, the components are simultaneously exposed to several conditions which normally are difficult to simulate. In many cases, the combined effect of being exposed to all of the environmental elements at once would provide insights into ruggedization for future space applications. The details of MISSE 1 to MISSE 6 missions can be found at NASA's MISSE webpage [2]

\section{SALIENT FEATURES OF THE MISSE 7 MISSION}

MISSE-7 is a suite of experiments that include over 700 new and affordable materials. The selected MISSE 7 specimens also have potential use in advanced systems for space applications. For MISSE-7 there are two PECs, 7A and 7B, which will be mounted on the outside of the ISS and hold samples on both sides of the PECs. PEC 7A's orientation will be zenith/nadir (space facing/Earth facing) while PEC 7B will face ram/wake (forward/backward) relative to the ISS orbit

Primary Responsibilities of MISSE 7A and 7B were carried out by Naval Research Laboratory and Air Force Research Laboratory, respectively. Glenn Research Center managed the MISSE 7 mission. NASA LaRC and Boeing played key roles. Besides above agencies, industry collaborators, and academia had experiments on MISSE 7B. NASA LaRC provided a flight worthy package with laser and lidar components along with other logistics support. This effort in NASA LaRC was a follow-on effort to MISSE 6 mission [3].

Figure 1 shows the laser and lidar components that were sent on MISSE 7 missions. The flight worthy package included 1.5 micron Fiber Laser, coherent receiver, several laser gain media, acousto-optic tunable filter, acousto-optic modulator, electro-optic modulator, and high power laser diode bar. NASA LaRC is involved with Active Sensing of CO2 over Nights, Days and ASCENDS and other lidar programs. ASCENDS is a mid-size, tier II future NASA mission [4]. Currently, several of these components are being used in ASCENDS related airborne and field test campaigns. These components have potential to be incorporated into future space based instrumentation based on their space qualification tests. 


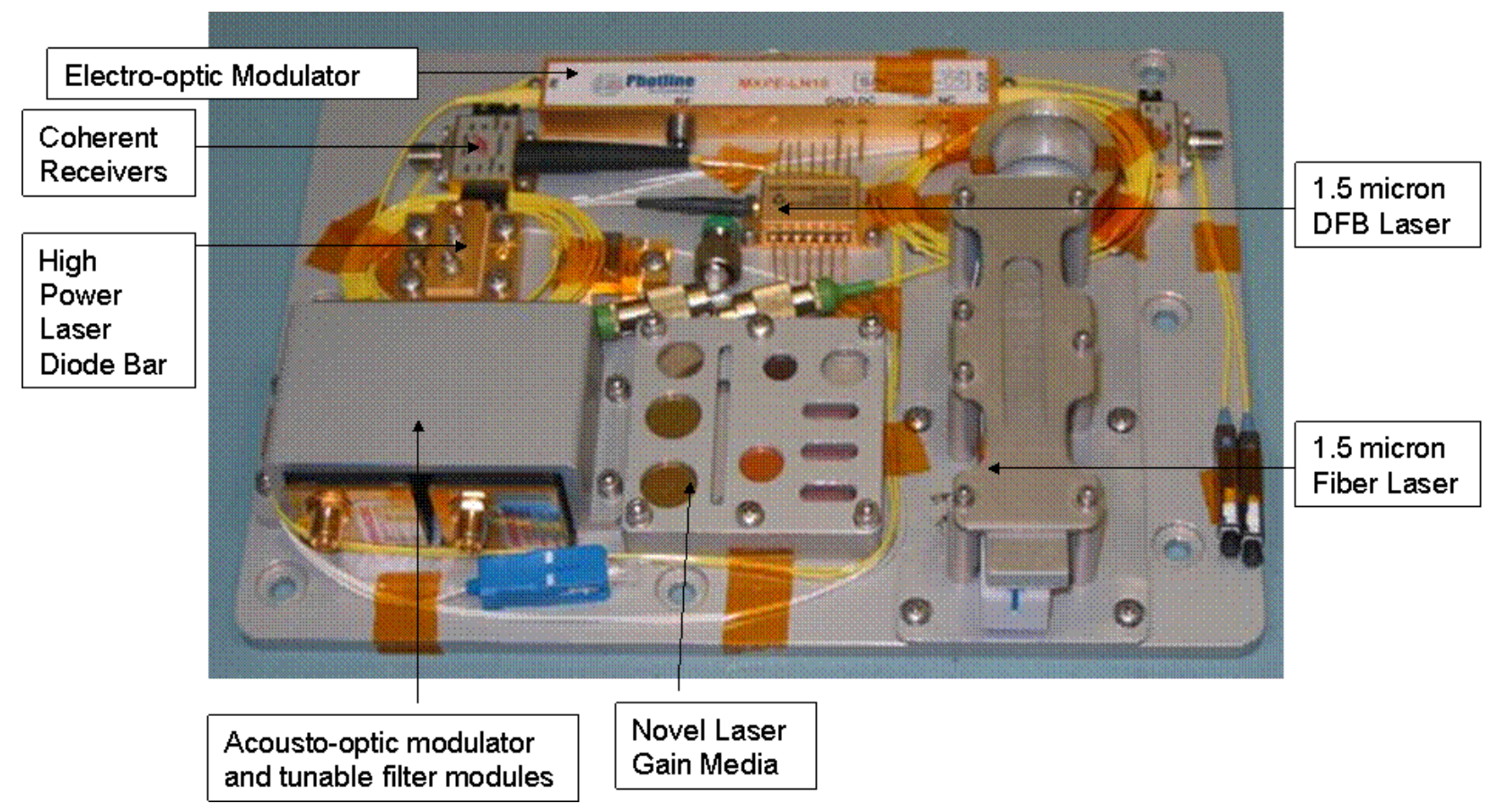

Figure 1. Laser and Lidar components mounted on a flight worthy package that was sent by NASA LaRC.

\section{MISSE 7 LAUNCH AND RETURN}

MISSE-7 was mounted to the ISS's exterior on an EXPRESS Logistics Carrier (ELC). The power and data were provided by the ISS. It used the ISS communication system uplink/downlink capabilities to receive commands downlink data. For this, no crew interaction was required. Prior to launch, the ExPRESS Payload Adapter (ExPA )platform on which the PEC were mounted as shown in Figure 2.

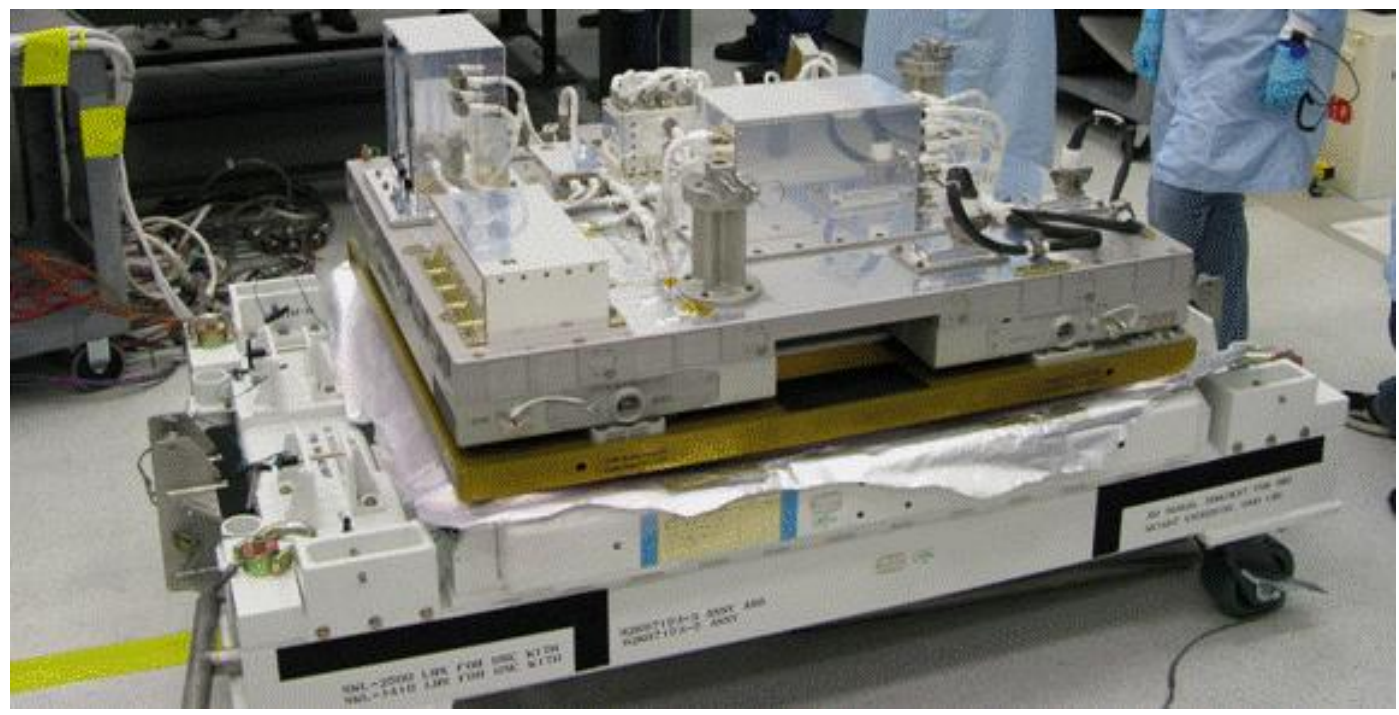

Figure 2. The ExPRESS Payload Adapter (ExPA )platform on which the PECs were mounted. 
MISSE-7 was launched on Space Shuttle mission STS-129. MISSE 7A \& \&B were attached outside the ISS on the EXPRESS Logistics Carrier 2 (ECL2) on the S3 truss, and then opened and exposed to space during a spacewalk on November, 23, 2009. MISSE 7 was retrieved by spacewalking astronauts on STS 134 on early morning hours of May 20, 2011, PEC 7A and PEC 7B from their external operating location on the ISS and transferred to the STS-134 Shuttle payload bay. Further details on the shuttle missions related to MISSE missions can be found in references [5-8].

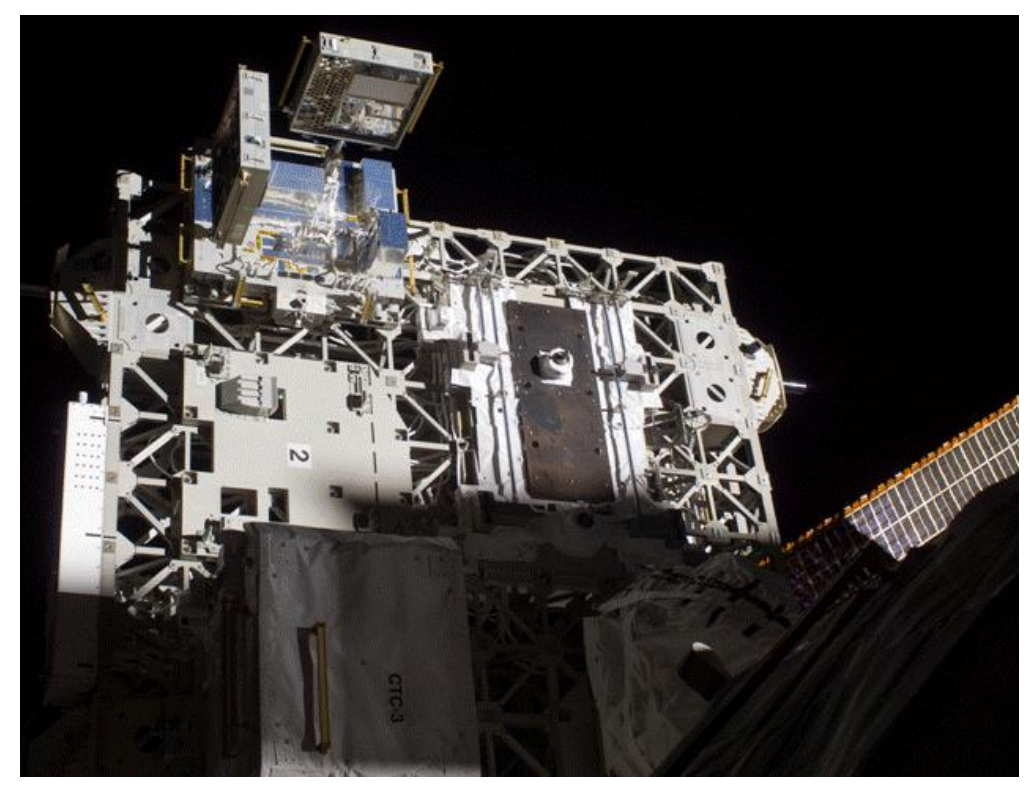

Figure 3. MISSE 7 on orbit.

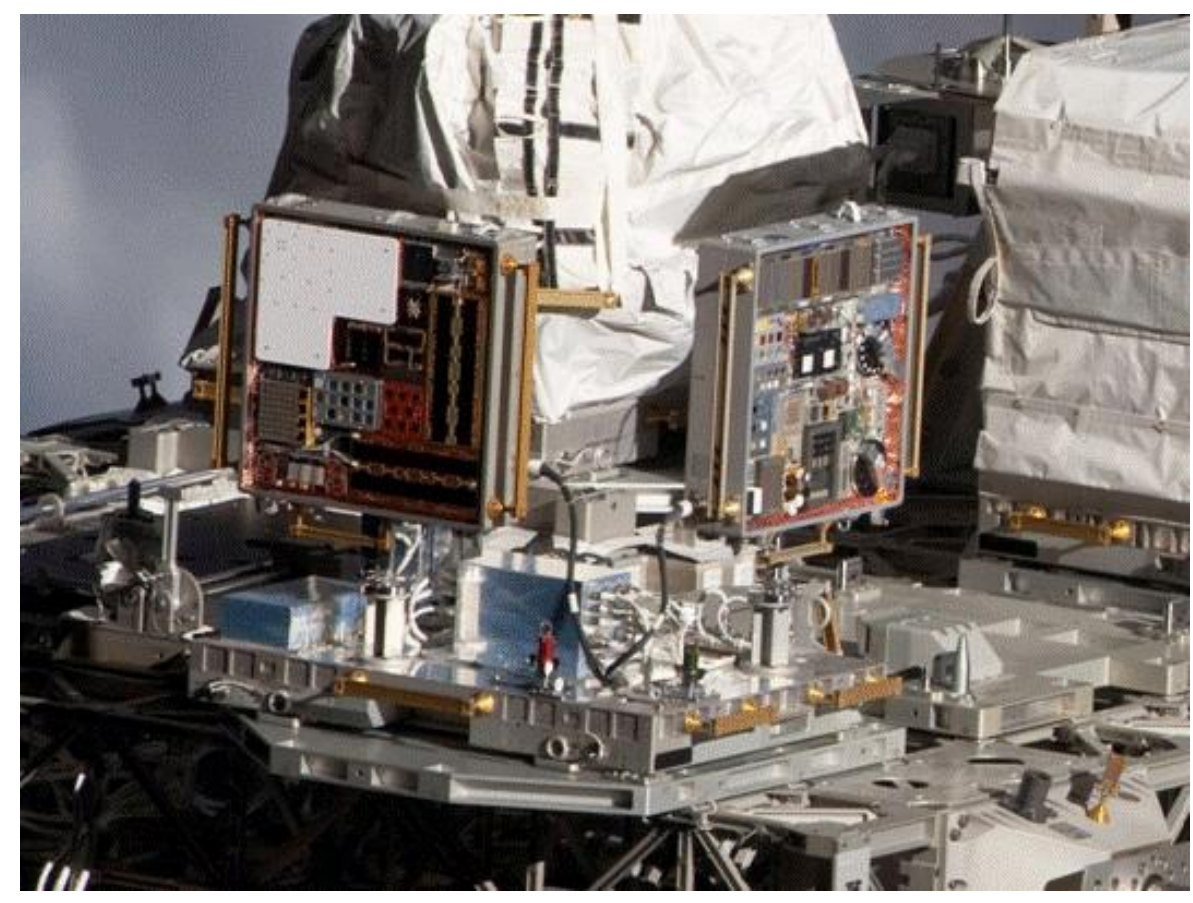

Figure 4. Zenith tray of MISSE 7A and Ram Tray of MISSE 7B. Photograph taken on 2/19/2010. 

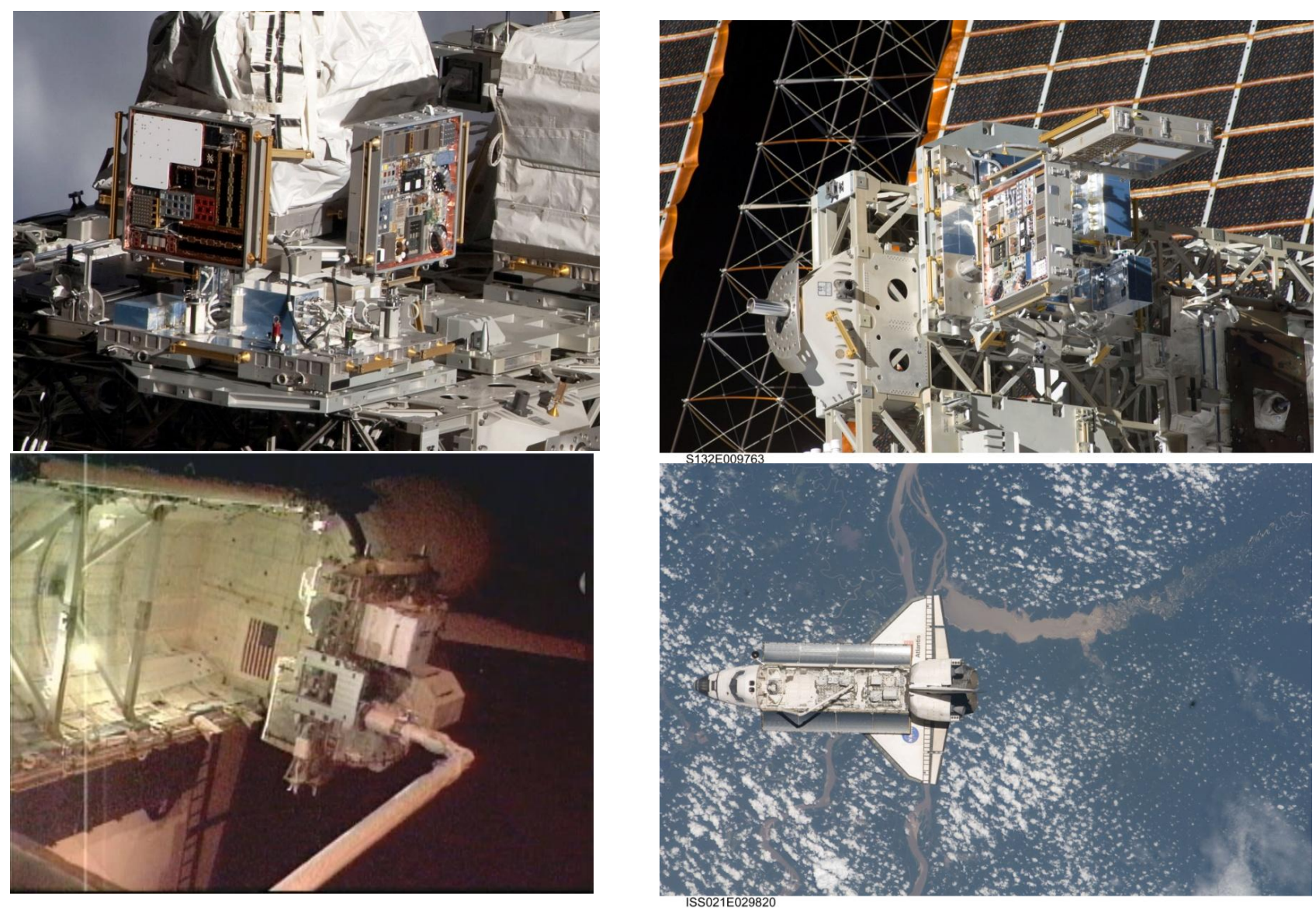

Figure 5. On-orbit pictures of MISSE 7.

\section{RETRIEVAL OF MISSE 7 PEC}

During its 1.5 year on-orbit mission, MISSE-7 has tested a variety of next-generation fiber lasers, solar cells and electronic devices and provided real-time downlink of science data. MISSE-7 has also continuously exposed cuttingedge material samples that will be analyzed in ground laboratories to determine how well they survived the space exposure effects of atomic oxygen, ultraviolet exposure, particle irradiation, and extreme temperature cycles. The onorbit pictures of MISSE 7 taken by space shuttle crew are shown in Figures 3-5. The retrieved MISSE 7 package was disassembled at Marshall Space Flight Center. In November, 2011, the test unit from LaRC was returned for further testing. Post-flight testing of individual components on this package is now underway. In Figure 6, the returned unit along with the detector and the EO modulator modules are shown. From visual inspection of the package, it appeared that the entire package remained unaffected by space exposure except for fading of label marking and disfigurations of a coating on a substrate. The comprehensive testing of the EO modulator is being planned. In the following section, preliminary results of performance testing of the single mode fiber coupled seed laser diode module are discussed. 

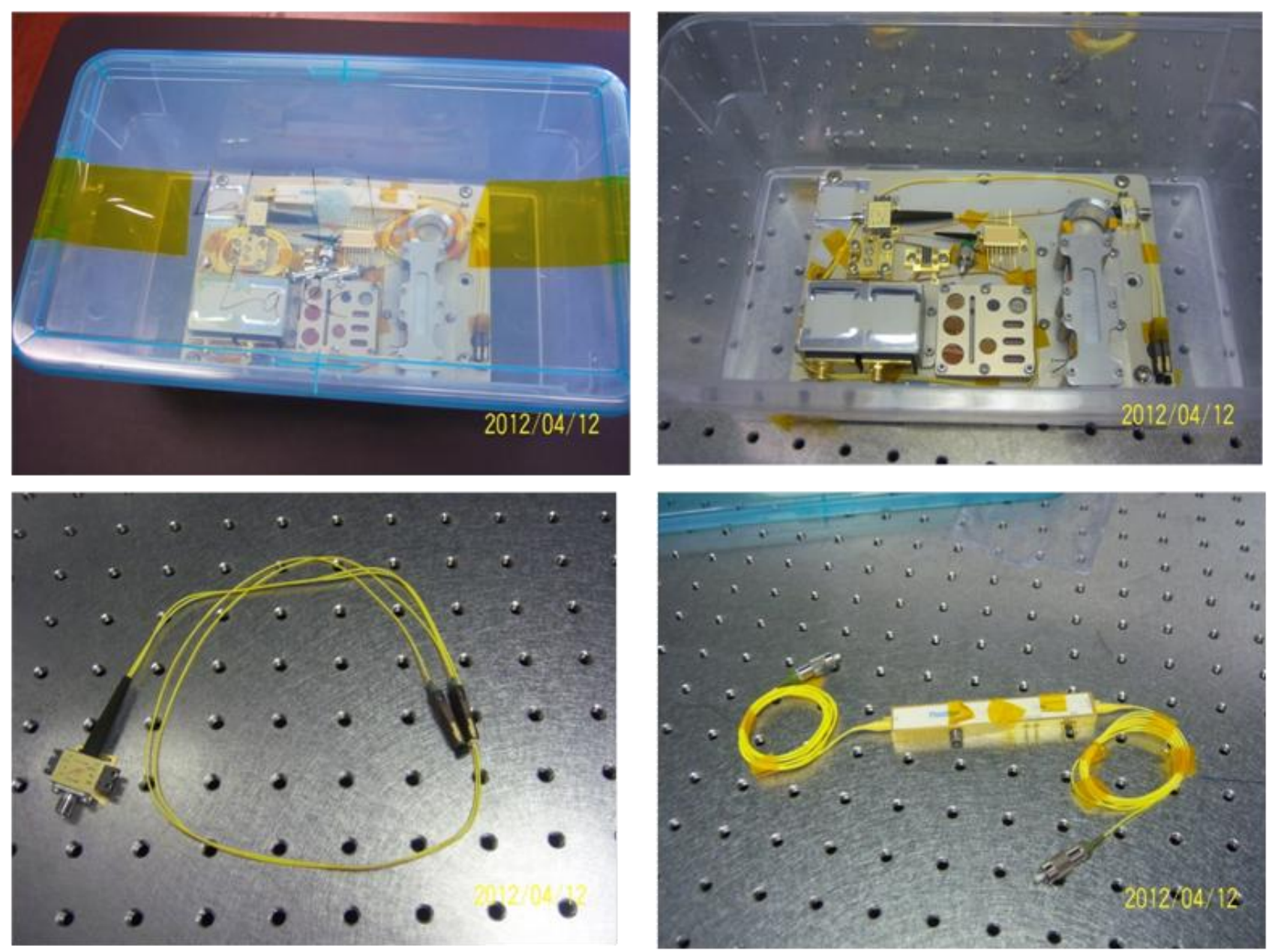

Figure 6. Returned package for post-flight tests. Top: Received package. Bottom Left: Balanced Receiver. Bottom Right: Electrooptic modulator.

\section{PRELIMINARY RESULTS OF LASER DIODE PERFORMANCE}

The laser diode module that was investigated was EM253-080-074. This single mode fiber coupled seed laser module was manufactured by EM4, Inc., now under the management of Gooch and Housego. It operated around a wavelength of 1.56 microns. This seed laser diode architecture is capable of providing wavelengths for $\mathrm{CO}_{2}$ sensing. This seed laser diode module technology was of interest to the NASA's ASCENDS related efforts for $\mathrm{CO}_{2}$ sensing investigations. For the purposes of experimental integrity, the laser diode tests were conducted at the EM4, Inc. lab facilities. The nominal chip temperature and output powers were $27^{\circ} \mathrm{C}$ and $80 \mathrm{~mW}$, respectively. The output power testing was carried out beyond $100 \mathrm{~mW}$. Table 1 shows a few pertinent specifications of this diode module. This unit was the earlier version of AA1401 device that replaces EM253 with minor changes in 2008. In the current AA1401 module, the case is electrically floating relative to the internal components. The datasheets of this laser seed module is available on the EM4, Inc. website [9].

\section{Diode Preparation and Test Procedure:}

The single mode fiber was broken while removing the device from the base plate attached by adhesive tapes. Since the fiber had been broken, we stripped a short section with a thermal stripper and cleaved the fiber with a flat cleave. The bare fiber was installed into an Agilent FC Bare Fiber adapter and connected to an Agilent 81625A power meter. The laser was installed into an ILX Lightwave LDM-4980 mount and the laser package and chip temperature were held 
at $25{ }^{\circ} \mathrm{C}$ using separate ILX Lightwave temperature controllers. The laser drive current was swept using an ILX Lightwave laser diode current source. The drive current was stepped in $10 \mathrm{~mA}$ increments and the current, voltage, and BFM photocurrent were recorded from the current source instrument, the power was recorded from the Agilent meter.

Table 1. General information of the laser diode module

\begin{tabular}{|l|c|c|c|c|}
\hline Lot Number & MO-05048 & & & \\
\hline Serial Number & E0028070 & & & \\
\hline Type & EM253-080-074 & & & \\
\hline Chip Temperature & 27 & ${ }^{\circ} \mathrm{C}$ & & \\
\hline Operating Power & 80 & $\mathrm{~mW}$ & & \\
\hline & & & & \\
\hline & Value & SpecMin & SpecMax & Unit \\
\hline Operating Current & 357 & 250 & 500 & $\mathrm{~mA}$ \\
\hline Dark Current & 0 & - & 2 & $\mu \mathrm{A}$ \\
\hline Monitor Current & 465 & 10 & 2500 & $\mu \mathrm{A}$ \\
\hline PER & 22 & 17 & & $\mathrm{~dB}$ \\
\hline Optical Frequency & 192.350 & 192.345 & 192.355 & $\mathrm{THz}$ \\
\hline Optical Wavelength & $1.55966 \mathrm{E}-06$ & $1.5597 \mathrm{E}-06$ & $1.56 \mathrm{E}-06$ & $\mathrm{~m}$ \\
\hline
\end{tabular}

\section{RESULTS AND DISCUSSIONS}

Figures 7 to 9 illustrate measured laser diode output power, drive current, monitor current and voltage characteristics. Figure 7 illustrates output power vs. drive current characteristics. Output power performance tests yielding greater than $100 \mathrm{~mW}$ were carried out. Post-flight measurements excellently followed pre-flight tests. Both traces are almost linear and appear to be overlapping on another without marked differences.

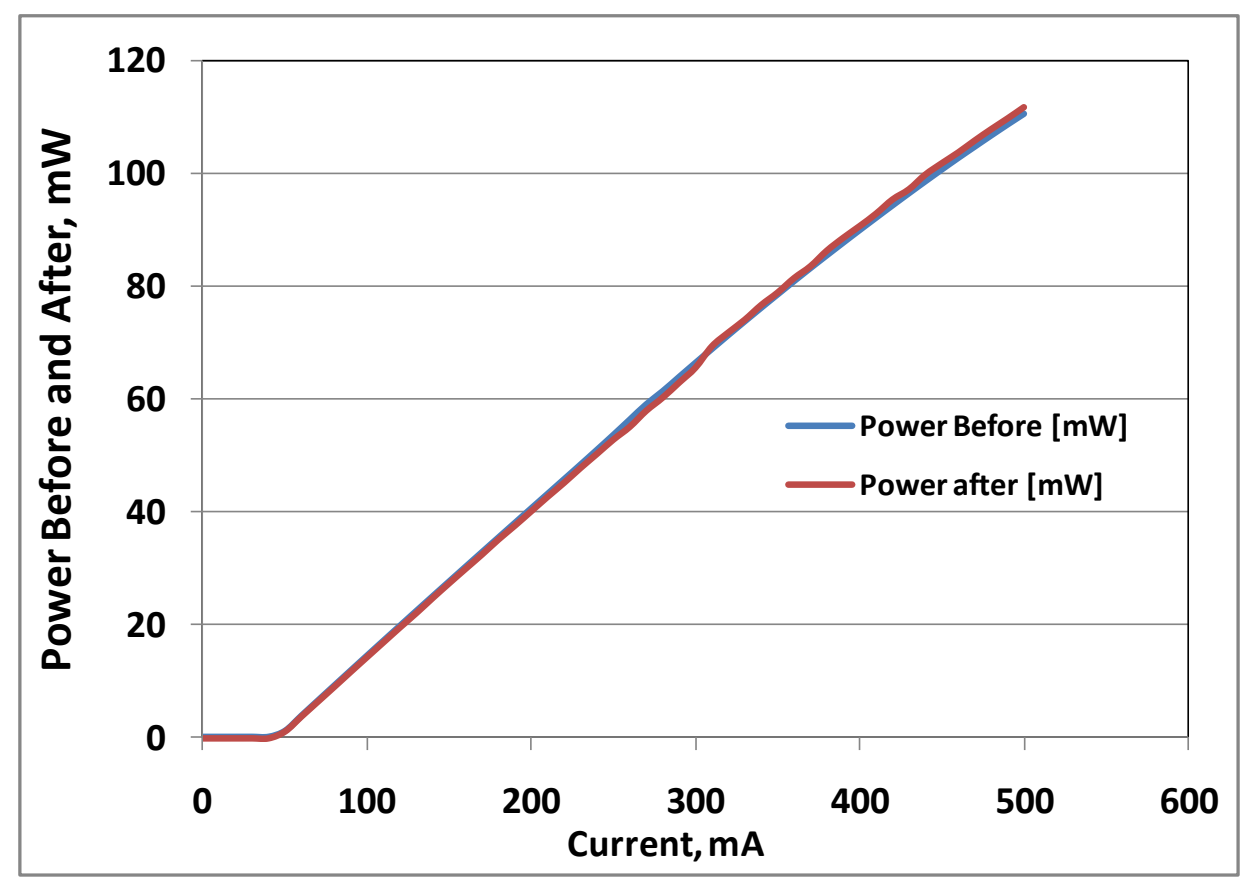

Figure 7. The drive current vs. power (LI) curve. Pre- and post-flight output power performance. No significant changes can be seen. 
Figure 8 shows the same characteristics along with monitor currents. Back facet and direct monitor current characteristics are plotted. One can see changes in monitor currents between 200 and $320 \mathrm{~mA}$ drive currents. The discontinuity is attributed to mode hopping. However, after $320 \mathrm{~mA}$ of drive current, the monitor current characteristics follow each other without any significant deviations. The values before and after the discontinuity are virtually identical. The different values are almost certainly test related since there is always some hysteresis in these measurements. So, the only real observable changes are that the fiber jacket became discolored (yellowish brown) and brittle after MISSE. Also, the ink on the cover label has completely disappeared.

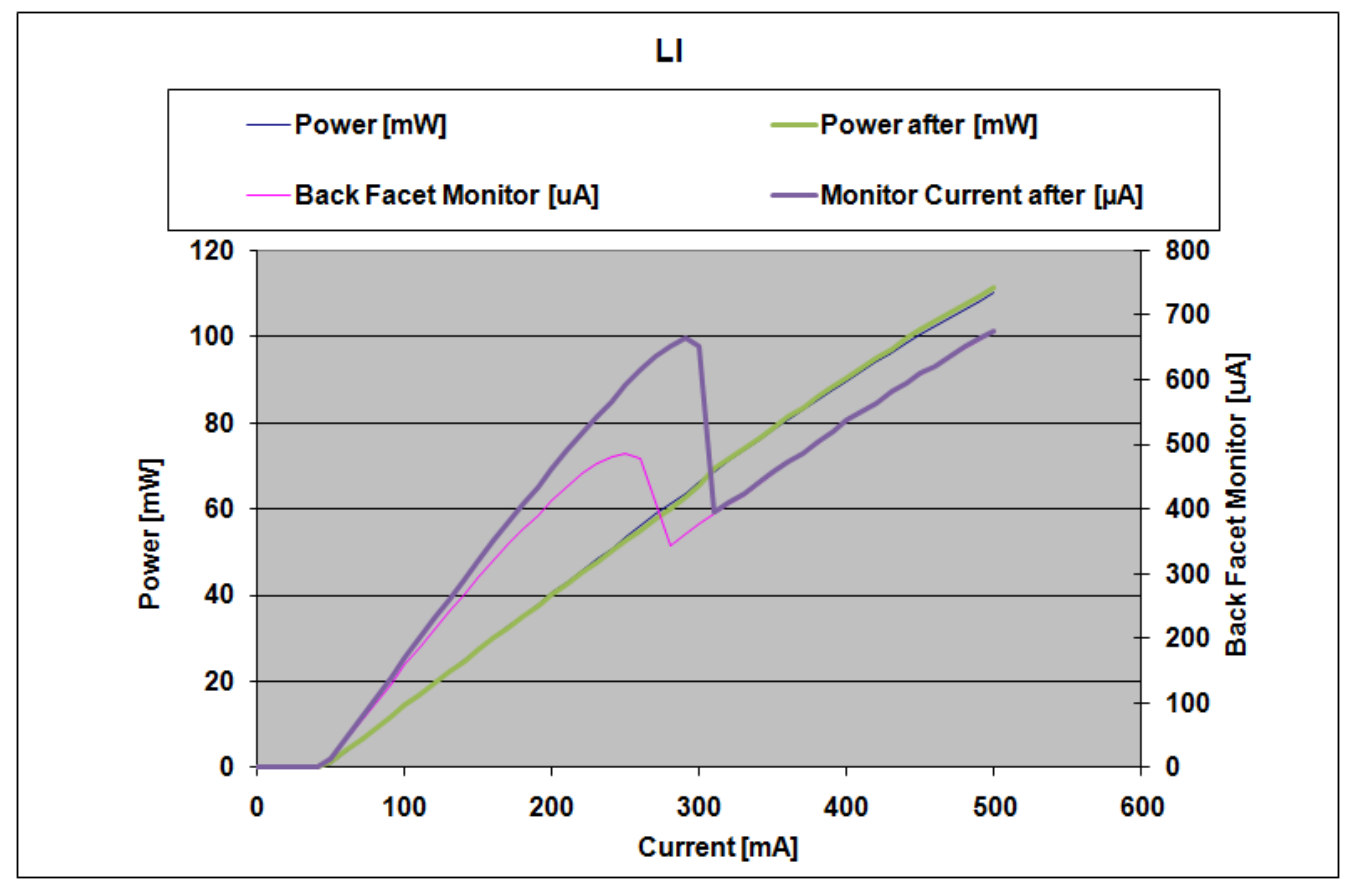

Figure 8. Ouput power characteristics are shown along with back facet and monitor currents. The differences in current characteristics is due to mode hopping. Monitor currents show no change after around $320 \mathrm{~mA}$ drive current and subsequently, are almost linear with output power.

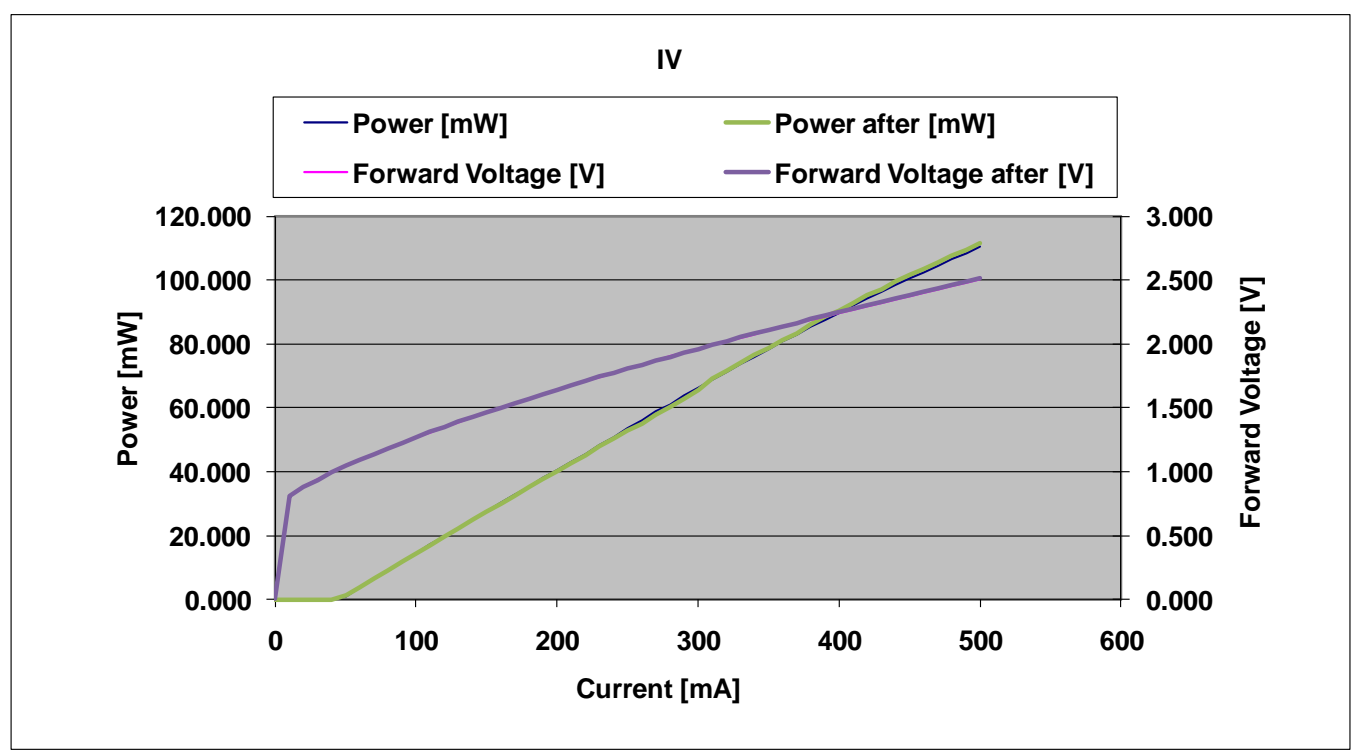

Figure 9. The diver current vs. voltage curves. Output power characteristics are also shown. The forward voltage characteristics before and after flights shown no significant changes. 


\section{SUMMARY AND CONCLUSIONS}

The objective of the MISSE program is to study the performance, stability, and long-term survivability of novel materials when subjected to the synergistic effects of the harsh space environment. So far, seven MISSE missions have been completed. The MISSE 7 mission was launched in November 2009 and returned to the Earth in May 2011 on STS 134. Several specimens and devices for use in Laser and Lidar systems for potential use in ASCENDS and other future NASA missions were selected. These specimens will undergo similar tests to look into deviations from pre-exposure characteristics. In this paper, laser diode tests are discussed. No significant deviations in the output power characteristics were observed. Over the next few months, we will be studying other laser diode characteristics for any

significant deviations from preflight tests. This data will allow us to design even better and rugged laser diode modules for future space qualification tests.

\section{ACKNOWLEDGMENTS}

The author acknowledges William $\mathrm{H}$. Kinard for providing guidance and support in the initial stages of Misse 7 mission development at NASA LaRC. The author acknowledges test support provided by Steve Coleman and Alex Rosiewich of EM4, Inc. Finally, the author thanks Karen Gibson and Terry Clark for mechanical engineering support.

\section{REFERENCES}

[1] William H. Kinard, "MIR Environmental Effects Payload (MEEP) Archive System," NASA, Langley Research Center, Hampton, Virginia. (http://setas-www.larc.nasa.gov/meep/meep.html and http://www.nasa.gov/centers/langley/news/factsheets/misse_2005.html).

[2] The NASA MISSE website: http://misseone.larc.nasa.gov/.

[3] Narasimha S. Prasad and William H. Kinard, "MISSE 6-Testing Materials in Space," Proc. SPIE 7095, 7095OD (2008).

[4] "Active Sensing of $\mathrm{CO}_{2}$ Emissions over Nights, Days, and Seasons (ASCENDS) Mission", NASA Science Definition and Planning Workshop, July 23-25, 2008, Univ. of Michigan, Ann Arbor, MI, available at http://decadal.gsfc.nasa.gov/documents/12-30-08-ASCENDS Workshop.pdf

[5] STS-123 MCC Status Report \#15 (http://www.nasa.gov/mission_pages/shuttle/shuttlemissions/sts123/news/STS123-15.html).

[6] STS-123 MCC Status Report \#25 (http://www.nasa.gov/mission_pages/shuttle/shuttlemissions/sts123/news/STS123-25.html).

[7] http://www.nasa.gov/mission_pages/station/science/misse2009.html.

[8] http://www.nasa.gov/mission_pages/station/science/misse.html.

[9] For laser diode datasheets, please see http://www.em4inc.com/sites/default/files/EM4\%201550\%20nm\%20High\%20Power\%20DFB's.pdf 